\title{
Philosophiques
}

\section{Jacques Bidet, Théorie de la modernité. Suivi de : Marx et le marché, Paris, PUF (coll. "Questions »), 1990, 320 p.}

\section{Gilles Labelle}

Volume 23, numéro 2, automne 1996

URI : https://id.erudit.org/iderudit/027413ar

DOI : https://doi.org/10.7202/027413ar

Aller au sommaire du numéro

Éditeur(s)

Société de philosophie du Québec

ISSN

0316-2923 (imprimé)

1492-1391 (numérique)

Découvrir la revue

Citer ce compte rendu

Labelle, G. (1996). Compte rendu de [Jacques Bidet, Théorie de la modernité.

Suivi de : Marx et le marché, Paris, PUF (coll. « Questions »), 1990, 320 p.]

Philosophiques, 23(2), 449-451. https://doi.org/10.7202/027413ar d'utilisation que vous pouvez consulter en ligne.

https://apropos.erudit.org/fr/usagers/politique-dutilisation/ 
Jacques Bidet, Théorie de la modemité. Suivi de : Marx et le marché, Paris, PUF (coll. "Questions "), 1990, 320 p.

L'ouvrage de Jacques Bidet s'inscrit dans le cadre des nouvelles lectures de l'œuvre de Marx paradoxalement suscitées par la disparition du mouvement communiste en Occident et l'effondrement des régimes communistes en Europe centrale et orientale. "Vaste chantier ", cette œuvre n'a désormais de sens que d'étre intégrée à la philosophie politique et à la théorie sociale modernes, selon l'auteur (p. 7). Car le travail de Marx souffre d'incomplétude en ceci qu'il rate la * matrice métastructurelle de la modernité " pour ne s'en tenir qu'à un possible de cette dernière, soit la structure capitaliste.

gue faut-il entendre par "matrice métastructurelle de la modernité "? C'est à cette question que cherche à répondre la première partie de l'ouvrage ("Théorie de la modernité ". p. 16-148). Ce qui définit le propre des temps modernes est, selon l'auteur, la contractualité, c'est-à-dire le fait que toute institution se représente étayée sur l'interindividualité. D'où trois strates de contractualité : une première, fondée sur la prolifération de contrats privés : une seconde, qui suppose ce que l'auteur nomme la "centricité ", fondée sur l'existence d'un contrat social ; et une troisième, fondée sur le regroupement volontaire de contractants placés dans une situation apparentée. Ces trois strates de contractualite sont indissociables les unes des autres. Il ne peut. en effet, y avoir de contrats privés entre individus (entre vendeurs et acheteurs, par exemple) que si sont foumies des garanties quant au respect des engagements pris. Autrement dit, les contrats privés supposent nécessairement un centre capable de sanctionner les contrevenants eventuels, lequel ne peut exercer ce rôle que s'il est lui-mème fondé sur un contrat auquel adhèrent la totalité des citoyens. Ce qui ne veut pas dire que le centre ne puisse étre conçu autrement que comme un "garant "des contrats privés. Le rapport de la contractualité centrale aux contrats privés est en fait déterminé par la troisième strate de contractualité, plus précisèment par l'effort des différentes associations afin de modeler le comportement du centre. C'est pourquoi les temps modernes sont le lieu d'un débat incessant et de conflits à propos des rapports qui doivent s'établir entre les trois strates de contractualité.

La matrice métastructurelle de la modemité est ainsi porteuse de plusieurs possibles selon l'auteur. Deux se sont réalisés jusqu'à maintenant. Le premier est désigné par le terme de capitalisme. Celui-ci constitue une structure dans laquelle le centre est considéré second par rapport aux contrats privés et en conséquence comme un simple garant de ces derniers. Pour que cet arrangement soit possible, il faut que les contrats privés soient représentès comme " naturels " et équitables, ce qu'énonce le libéralisme. En réalité, comme Marx l'a bien montré, ces rapports sont inéquitables, de telle sorte que l'on peut avancer que le libéralisme correspond aux intérêts des groupes qui tirent avantage de cette inéquité, soit les propriétaires des moyens de production. Le communisme représente un autre arrangement possible des éléments qui composent la métastructure de la modernité. Contrairement à ce que Marx pouvait penser, ce nouvel arrangement ne marque pas la fin de la "préhistoire " de l'humanité et l'ouverture d'un tout nouveau rapport de cette dernière à elle-même et aux choses. Le communisme consiste en fait essentiellement en une inversion des rapports entre contrats privés et contractualité centrale : le centre y étant posé comme détenant un primat sur la première strate de contractualité, il la détermine et la régit. Ce qui, par ailleurs, ne conduit pas nécessairement à la destruction totale des contrats privés (du marché) et à la reconstruction à partir du centre des rapports 
sociaux (le plan comme antithèse absolue du marché). Car Marx et les marxistes estiment que le marché gènère spontanément un ensemble de rapports stables, lesquels constituent déjà l'embryon d'un plan. De ces rapports, il s'agit seulement d'éliminer le póle constitué par le groupe des capitalistes, remplacés par des délégués du centre, dêsormais confondu (en principel avec l'association des producteurs non propriétaires des moyens de production.

C'est ce deuxième possible de la métastructure de la modernitè, gangrené par la bureaucratie et l'absence de démocratie, l'autonomie de la troisième strate de contractualité s'y trouvant éliminée, qui se fissure maintenant. Faut-il pour autant en revenir au premier possible défini par le capitalisme? Pour l'auteur il s'agit plutôt de penser ce que peut représenter "le socialisme dans la modernité " (p. 95 ss). Au rebours à la fois de Kant, qui dissocie l'être et le devoir-être, et de Hegel, qui fait découler le second du premier, il faut penser un possible inscrit dans la matrice de la modernité mais dont rien ne garantit l'avènement. Les philosophies politiques de Habermas et de Rawls, à condition d'ètre revues à la lumière de la théorie de la modernité précédemment exposée ou de la critique marxienne du capitalisme, peuvent aider à penser ce possible socialiste. De Habermas il faut retenir la critique de la rationalité instrumentale et l'effort pour conceptualiser l'agir communicationnel orienté en vue d'un consensus, conditions indispensables à l'établissement de la raison démocratique. Car celle-ci est seule à mèrne de permettre létablissement de rapports réflèchis entre les trois strates de contractualité. Il ne s'agit ni de postuler que les contrats privés doivent ètre soumis au centre ou l'inverse; mais bien de poser que les rapports qui doivent s'établir dans chacune des strates et entre elles ne peuvent ètre déterminés qu'au travers de la discussion publique. Ce pourquoi il faut "corriger "Habermas, qui a tendance, en définitive, à séparer les ensembles dits systémiques (le marché, l'administration) du monde vécu, et ainsi à aboutir à un dualisme entre nécessité et liberté (p. 107-109). De Rawls. il faut retenir le premier principe qui doit présider à la vie de la Cité, suivant lequel les libertês doivent ètre partagées par tous les citoyens, ce qui paraît, ici encore, une condition de la raison democratique. Mais il faut également refuser le " préjugé libéral * qui, selon l'auteur, conduit Rawls à considérer ce principe en quelque sorte indépendamment du second, suivant lequel les différences sont acceptables à la condition, notamment, de contribuer à l'amélioration du sort des plus défavorisés. Car en l'absence d'une réduction significative des inégalités socio-èconomiques entre individus et associations, l'exercice par tous des libertés publiques perd une grande partie de son sens dans nos sociètés (p. 127, 135).

La seconde partie de l'ouvrage (" Marx et le marché ", p. 149-272) constitue une analyse de lincapacité de Marx à saisir la matrice métastructurelle de la modernitê. En raison de sa volonté de "démystifier " la contractualité mise en évidence par les auteurs libéraux, Marx en vient à traiter celle-ci à la seule lumière du rapport social capitaliste. Par une lecture serrée, l'auteur montre que si Marx est plus sensible à l'autonomie du marché à l'égard du capital dans le Capital que dans les Grundrisse, il persiste à considérer la contractualité comme un phénomène dont le sens est révélé en définitive par l'analyse des rapports de classes. Conséquemment, le centre, le pouvoir politique, n'est pas pour Marx un lieu où peut se déployer la raison démocratique et ainsi se décider des rapports autres entre les différentes strates de contractualité, mais bien un - instrument de la classe dominante . Par là, selon l'auteur, Marx se situe en retrait de Hegel, lequel, refusant les théories du contrat social qu'il estime fondées dans l'interindividualité générant les contrats privés, reconnait cependant l'existence d'un niveau "social-communautaire "devant dépasser- 
conserver les rapports établis sur le marché. S'il se rend ainsi partiellement aveugle à la structure capitaliste, Hegel saisit néanmoins les éléments principaux de la métastructure de la modernité : Marx, en réaction, fait l'inverse (p. 275 ss).

Louvrage de Jacques Bidet a un statut ambigu, nous semble-t-il. La deuxième partie, qui aurait peut-ètre dũ ètre présentée d'abord (puisqu'il y est question des limites du projet de Marx), se rattache principalement à l'histoire des idées. L'auteur se fait exégète et montre, avec une richesse de détails dont la présente recension ne rend certes pas compte, l'évolution de la pensée de Marx des Grundrisse au Capital. Tout etudiant de l'œuvre de Marx pourra y trouver de quoi alimenter sa réflexion. La première partie se situe à un autre niveau. Lauteur veut y développer ce qu'il nomme un " métamarxisme * qui, comme on l'a indique ci-dessus, constituerait une sorte de synthèse de ce qui est valable chez Marx et de certaines philosophies politiques ou théories sociales contemporaines. Guoique son objectif ne soit pas dénué de pertinence - on peut, en effet, raisonnablement penser que l'œuvre de Marx a tout à gagner d'une mise en rapport avec d'autres et d'un dialogue avec elles - la montagne nous parait accoucher ici d'une souris. Car le projet socialiste que l'auteur décrit revient, en définitive, à un appel à revigorer la raison démocratique dans nos sociétés (d'où le recours à Habermas et Rawls). Au travers de la discussion publique, est-il présumé. les citoyens pourront décider des rapports à établir entre les différents niveaux de contractualité. Mais en quoi l'exercice de la raison démocratique conduira-t-il nécessairement à décider, par exemple. la réduction des inégalités dans la première strate de contractualité et l'exercice actif de la souveraineté du peuple dans la seconde ? S'il s'agit de repenser la démocratie noble tāche, nous en convenons - aussi bien s'en tenir à une réflexion sur les conditions qui la rendent possible: point n'est besoin d'invoquer un "projet socialiste " dont les assises demeurent ici, somme toute, assez vagues.

Gilles Labelle

Département de science politique Université d'Ottawa 